\title{
ALMOST ISOMORPHISM FOR COUNTABLE STATE MARKOV SHIFTS
}

\author{
MIKE BOYLE, JEROME BUZZI, AND RICARDO GOMEZ
}

Dedicated to Peter Walters and Klaus Schmidt, on the occasion of their sixtieth birthdays.

\begin{abstract}
Countable state Markov shifts are a natural generalization of the well-known subshifts of finite type. They are the subject of current research both for their own sake and as models for smooth dynamical systems. In this paper, we investigate their almost isomorphism and entropy conjugacy and obtain a complete classification for the especially important class of strongly positive recurrent Markov shifts. This gives a complete classification up to entropyconjugacy of the natural extensions of smooth entropy-expanding maps, e.g., $C^{\infty}$ smooth interval maps with non-zero topological entropy.
\end{abstract}

\section{Contents}

1. Introduction

2. Definitions and background 3

3. Magic words, almost isomorphism and entropy-conjugacy 5

4. Zeta functions 8

5. The Loops Lemma 10

6. Main Results 14

7. Application to other dynamical systems 17

8. Remarks 18

$\begin{array}{ll}\text { References } & 20\end{array}$

\section{INTRODUCTION}

In this paper, "Markov shift" means a countable (possibly finite) state irreducible Markov shift. We use the same symbol to denote the domain of a Markov shift and the shift map on this domain. There are several characterizations (2.3) of the class of strongly positive recurrent (SPR) Markov shifts; this is the class of Markov shifts which most resemble finite state Markov shifts.

A map $\varphi: S \rightarrow T$ between Markov shifts is a one-block code if there is a function $\Phi$ from the symbol set of $S$ into the symbol set of $T$ such that $(\varphi x)_{n}=\Phi\left(x_{n}\right)$, for

2000 Mathematics Subject Classification. Primary: 37B10; Secondary: 37B40, 37C99, 37 D35.

Key words and phrases. entropy; countable state Markov shift; topological Markov chain; almost isomorphism; entropy conjugacy; magic word; strong positive recurrence; Artin-Mazur zeta function; smooth ergodic theory.

The research of J. Buzzi was also supported by the Center for Dynamical Systems of Penn State University and the kind hospitality of the University of Maryland. 
all $x$ and $n$. A $T$-word $W$ (of length $|W|$ ) is a magic word for such a map $\varphi$ if the following hold.

(1) If $y \in T$ and $\{n \in \mathbb{Z}: y[n, n+|W|-1]=W\}$ is unbounded above and unbounded below, then $y$ has a preimage under $\varphi$.

(2) There is an integer $I$ such that whenever $C$ is a $T$-word and two points $x$ and $x^{\prime}$ of $S$ satisfy $(\varphi x)[0,2|W|+|C|-1]=W C W=\left(\varphi x^{\prime}\right)[0,2|W|+|C|-1]$, then $x[I, I+|W|+|C|-1]=x^{\prime}[I, I+|W|+|C|-1]$.

(In the constructions of this paper, the integer $I$ of the last condition will be zero.) It follows from (2) that the preimage in (1) is unique.

We define two Markov shifts $S$ and $T$ to be almost isomorphic if there exist another Markov shift $R$ and injective one-block codes $R \rightarrow S, R \rightarrow T$ each of which has a magic word (recall that all Markov shifts are understood to be irreducible). An almost isomorphism will induce a shift-commuting, Borel bimeasurable bijection between the images of the one-block codes, and thereby will induce isomorphisms of measurable systems $(S, \mu) \rightarrow(T, \nu)$ for shift-invariant Borel probabilities $\mu, \nu$ which assign these images measure 1 . The collection of such measures will include the ergodic measures which give positive measure to every nonempty open set, and in the SPR case will include all ergodic measures with entropy sufficiently close to the topological entropy (defined (2.1) as the supremum of the measure-theoretic entropies with respect to invariant Borel probabilities). These isomorphisms will be finitary (homeomorphisms between measure-one sets), with exponentially fast coding time for exponentially recurrent ergodic measures (such as the unique measure of maximal entropy, in the SPR case).

Our main result (6.4) is that SPR Markov shifts are almost isomorphic if and only if they have the same entropy and period. This can be viewed as an analogue of the Adler-Marcus classification of irreducible shifts of finite type up to almost topological conjugacy by entropy and period (8.2). The classification beyond SPR cannot possibly have the same simplicity (3.6). We also give a sufficient condition for almost isomorphism of not necessarily SPR Markov shifts (6.2).

Two systems are entropy conjugate if they are Borel conjugate after restriction to sets which have full measure for all ergodic measures with entropy near the topological entropy (see Definition 3.3). Various smooth, piecewise smooth and symbolic systems are known (Theorem 7.1) to have natural extensions which are entropy conjugate to a finite union of SPR Markov shifts. Consequently, our main result provides simple invariants which classify the natural extensions of these systems up to entropy conjugacy (Theorem 7.2).

One motivation of our paper is recent interest in the thermodynamic formalism for countable state Markov shifts $[10,17,22,23,24,38,48,49,50,52]$. In a companion paper [4], we describe a reasonable class of Borel potentials which behaves well under almost isomorphism. We are also motivated by the use of countable state Markov shifts to code some partially or piecewise hyperbolic systems $([5,8,9,25,26,27,29,30,56,57,59,64]$ and successors of [64]); investigations of coding relations among Markov shifts (see [12, 13, 14, 15, 16] and their references); and a longstanding finite state coding problem for Markov measures not of maximal entropy (8.4).

This paper is dedicated to Klaus Schmidt and Peter Walters, on the occasion of their sixtieth birthdays. Words from the poet E.E.Cummings [11] suit them: 
septembering arms of year extend

less humbly wealth to fool and friend

\section{DEFinitions AND BACKGROUND}

Let $A$ be a square matrix with nonnegative integer entries, with rows and columns indexed by a finite or countable set $\mathcal{I}$. View $A$ as the adjacency matrix of a directed graph with some set of edges $\mathcal{E}_{A}$. Give $\mathcal{E}_{A}$ the discrete topology; give $\left(\mathcal{E}_{A}\right)^{\mathbb{Z}}$ the product topology; let $S_{A}$ denote the set of doubly infinite sequences on $\mathcal{E}_{A}$ corresponding to walks through the graph; and let $S_{A}$ have the topology it inherits as a subspace of $\left(\mathcal{E}_{A}\right)^{\mathbb{Z}}$. The shift map homeomorphism on $S_{A}$ is defined by sending a bisequence $\left(x_{n}\right)$ to the bisequence $\left(y_{n}\right)$ such that $y_{n}=x_{n+1}$ for all $n$. Given a point/bisequence $x$, we let $x[i, j]$ denote the word $x_{i} x_{i+1} \ldots x_{j}$. If $W$ is a path of $n$ edges (or a finite sequence of $n$ symbols), then $|W|$ denotes $n$. We will use the same symbol (e.g. $S_{A}$ ) for the shift map and its domain. $S_{A}$ is uniquely determined by $A$, up to the naming of the edges in the graph; in this paper, we can safely ignore this ambiguity, and refer to "the" Markov shift defined by $A$. We use the edge shift presentation rather than the vertex shift presentation, but this paper could have been written entirely with the vertex shift presentation.

The matrix $A$ is irreducible if for every row $i$ and column $j$ there exists $n>0$ such that $A^{n}(i, j)>0$. (The term "indecomposable" as used in [24] is synonymous with our "irreducible".) Convention: in this paper, by a Markov shift we will mean a homeomorphism $S_{A}$ defined by a (finite or countably infinite) irreducible matrix $A$ over $\mathbb{Z}_{+}$.

By a graph we will always mean a directed graph, with finitely or countably infinitely many vertices and edges. Given a vertex $v$ in a graph, a first return loop to $v$ is a path of edges which begins and ends at $v$ and otherwise does not visit $v$. The number of edges in a path $p$ is its length, $|p|$. A loop graph (in [24], a petal graph) is a graph $\mathcal{G}$ with a distinguished vertex $v$, such that $\mathcal{G}$ is the union of the first return loops to $v$, and every vertex except $v$ lies on a unique first return loop. From here, for brevity by a loop in a loop graph we will always mean a first return loop to the distinguished vertex.

Let $f$ be a power series $\sum_{n=1}^{\infty} f_{n} z^{n}$ with nonnegative integer coefficients, i.e. $f \in z \mathbb{Z}_{+}[[z]]$. We will sometimes use the notation $\operatorname{rad}(f)$ to denote the radius of convergence of $f$. A loop graph $\mathcal{G}_{f}$ for $f$ is a loop graph which for each $n$ contains exactly $f_{n}$ loops of length $n$. The loop graph is uniquely determined by $f$ up to the naming of the edges and vertices. Given $\mathcal{G}_{f}$, the loop shift $\sigma_{f}$ is the Markov shift $S_{A}$ such that $A$ is the adjacency matrix of a loop graph for $f$.

Suppose $S_{A}$ is a Markov shift, with associated graph $\mathcal{G}(A)$, and $v$ is a vertex in $\mathcal{G}(A)$. Let $f_{n}$ denote the number of first return loops of length $n$ to $v$, and assume $f_{n}<\infty$ for all $n$ (a property which is independent of the choice of vertex). Let $\sigma_{f}$ be the corresponding loop shift. The period of $S_{A}$ (or of $\sigma_{f}$ ) is the g.c.d of $\left\{n: f_{n}>0\right\}$, and does not depend on the choice of vertex. $S_{A}$ is mixing if and only if the period is 1 .

Definition 2.1. By the entropy $h\left(S_{A}\right)\left(=h\left(\sigma_{f}\right)\right)$, we will mean the Gurevich entropy [21], which is the supremum of the measure theoretic entropies over invariant Borel probabilities. This entropy equals $\log \left(\varlimsup \lim \left|r_{n}\right|^{1 / n}\right)$, where $r_{n}$ is the number of loops (not necessarily of first return) at an arbitrary vertex, and the limsup here is a limit when $S_{A}$ is mixing. 
Let $\log (\lambda)$ denote the entropy of $S_{A}$ and suppose $0<\log (\lambda)<\infty$. Four basic classes can be described in terms of the sequence $\left(f_{n}\right)$.

(1) $S_{A}$ is transient if $\sum f_{n} / \lambda^{n}<1$

(2) $S_{A}$ is recurrent if $\sum f_{n} / \lambda^{n}=1$

(3) $S_{A}$ is positive recurrent if $\sum f_{n} / \lambda^{n}=1$ and $\sum n f_{n} / \lambda^{n}<\infty$

(4) $S_{A}$ is strongly positive recurrent (SPR) if $\varlimsup_{\lim }\left|f_{n}\right|^{1 / n}<\lambda$.

(We are using names of matrix classes to describe the Markov shifts they define.) Here $(4) \Longrightarrow(3) \Longrightarrow(2)$. The properties (1)-(4) don't depend on the particular choice of vertex [24]. The classes (1)-(3) correspond to the classical Vere-Jones classification $[60,61]$ of $\mathbb{N} \times \mathbb{N}$ nonnegative matrices. A finite entropy Markov shift is positive recurrent if and only if it has a measure of maximal entropy $\mu$ (i.e., $h(S)=h(S, \mu))$, in which case there is only one measure of maximal entropy [21].

Markov shifts defined over a countable alphabet may differ significantly from those which have a finite alphabet (subshifts of finite type), see e.g. [12, 24, 31, $50,52]$. The search for well-behaved Markov shifts leads to the class of SPR shifts. The notion of SPR already appeared in Vere-Jones' work [61] in the special case of stochastic matrices as a necessary and sufficient condition for some exponential convergence. Following work of Salama (see $[45,46,47]$ ) on conditions for the strict decrease of entropy in proper subsystems, the class of SPR shifts was introduced independently by Ulf Fiebig [19] and by Gurevich [22], who introduced the class of "stably recurrent" matrices, which contain those defining what we call the SPR Markov shifts. In [24], this class is developed further as the fundamental class of "stable positive" matrices [24]. We view "SPR" as also abbreviating "stable positive recurrent".

We will record some of the conditions on a Markov shift which are equivalent to SPR. For one, we first recall the definition of exponential recurrence while remarking some equivalences.

Remark 2.2. Suppose $(S, \mu)$ is an ergodic automorphism of a topological space with a Borel probability measure. Let $V$ be a measurable set such that $\mu V>0$. Let $r_{V}$ be the return-time function on $V, r_{V}(x)=\min \left\{n>0: S^{n} x \in V\right\}$. By ergodicity the system $(S, \mu)$ is isomorphic to a tower over $V$ with return time function $r_{V}$. It is not difficult to check that the following are equivalent:

(1) $(S, \mu)$ is exponentially recurrent: i.e. for every open set $V$ with $\mu V>0$,

$$
\varlimsup_{n}\left(\mu\left\{x \in V: r_{V}(x) \geq n\right\}\right)^{1 / n}<1 .
$$

(2) $(S, \mu)$ is exponentially filling: i.e. for every open set $V$ with $\mu V>0$,

$$
\varlimsup_{n}\left(\mu\left\{x \in S: x \notin \cup_{k=1}^{n} S^{-k} V\right\}\right)^{1 / n}<1 .
$$

(3) $\left(S^{-1}, \mu\right)$ is exponentially recurrent.

Proposition 2.3. The following are equivalent conditions on a Markov shift $S$.

(1) $S$ is SPR.

(2) Removing any edge from the graph defining $S$ strictly lowers the entropy ([19], [47],[24, Remark 3.16]; see [45]).

(3) Some (equivalently every) local zeta function (counting the number of fixed points $x \in[v]$ for some vertex $v$ ) has a non-trivial meromorphic extension (see [24] and Section 4). 
(4) $S$ has a measure of maximal entropy $\mu$, and $(S, \mu)$ is exponentially recurrent.

Proof. We will prove $(4) \Longleftrightarrow(1)$. Note that both conditions imply that there exists a measure of maximum entropy and recall that such a measure gives positive measure to every non-empty open subset [21]. We may and do assume that we have such a measure $\mu$.

Let $V_{I}$ be the open set of points $x$ such that the edge $x_{0}$ begins at a given vertex $I$. Let $f_{n}$ be the number of first return loops to $I$. By the specific form for $\mu[31,21]$, there is a constant $c_{I}>0$ such that, for any first return loop $W_{0} \ldots W_{n-1}$, the set $[W]:=\left\{x: x_{0} \cdots x_{n-1}=W\right\}$ has $\mu$-measure $c_{I}(1 / \lambda)^{n}$. Thus $\mu\left(S \backslash \cup_{n=1}^{N} S^{n} V_{I}\right)$ goes to zero exponentially fast, i.e. $V_{I}$ is exponentially filling in $(S, \mu)$, if and only if $\sum_{n=N+1}^{\infty} c_{I}(n-N)(1 / \lambda)^{n}$ goes to zero exponentially fast. But the latter is equivalent to the SPR property.

To conclude, it is enough to see that SPR implies not only exponential filling for the sets $V_{I}$ for any vertex $I$, but for all non-empty open sets $V$. Any such $V$ contains a cylinder set $C=\{x: x[-k, k]=W\}$ for some word $W$, and $W$ becomes a vertex in a higher block presentation $S^{\prime}$ of $S$. Because $S^{\prime}$ is again SPR [24, Propostion 2.12], $C$ is exponentially filling in $S^{\prime}$, and therefore so is $V$ in $S$.

For more background, see $[24,31,45]$ and their references for Markov shifts and nonnegative matrices; [63] for entropy and ergodic theory; and [31, 35] for symbolic dynamics.

\section{MAGIC WORDS, ALMOST ISOMORPHISM AND ENTROPY-CONJUGACY}

We assume the definitions already given in the Introduction. Given a subset $K$ of a Markov shift $S$, we let $\mathcal{M}(K)$ denote the set of shift-invariant Borel probabilities $\mu$ on $S$ such that $\mu K=1$. Let $K^{\prime}$ be a subset of another Markov shift $S^{\prime}$. Given a shift-commuting Borel automorphism $\gamma: K \rightarrow K^{\prime}$, we may use the same symbol $\gamma$ for related maps such as $\gamma: \mathcal{M}(K) \rightarrow \mathcal{M}\left(K^{\prime}\right)$. Recall a measure on a space has full support if it is nonzero on every nonempty open set. If $\mu$ is a shift invariant measure on $S$, then it defines a measure-preserving system $(S, \mu)$. An isomorphism of measure preserving shift systems $(S, \mu) \rightarrow\left(S^{\prime}, \mu^{\prime}\right)$ is a bimeasurable, shift-commuting, measure-preserving bijection from a subset $K$ of $S$ to a subset $K^{\prime}$ of $S^{\prime}$, where $\mu K=\mu^{\prime} K^{\prime}=1$. Such an isomorphism is finitary if $K$ and $K^{\prime}$ can be chosen so that the map $K \rightarrow K^{\prime}$ is a homeomorphism, with respect to the topologies $K$ and $K^{\prime}$ inherit as subsets of the topological spaces $S$ and $S^{\prime}$. In this case, for $\mu$-almost all $x$, there exists a minimal nonnegative integer $n=n(x)$ such that for $\mu$-almost all points $x^{\prime}$ with $x^{\prime}[-n, n]=x[-n, n]$, we have $(\varphi x)_{0}=\left(\varphi x^{\prime}\right)_{0}$. (In other words, $\mu$-almost surely $\varphi$ is a variable length block code.) We then define the expected coding time of $\varphi$ to be $\int_{S} n(x) d \mu$. We say $\varphi$ has exponentially fast coding if $\varlimsup_{k}|\mu\{x: n(x) \geq k\}|^{1 / k}<1$; this property implies the expected coding time is finite. We say systems $(S, \mu)$ and $\left(S^{\prime}, \mu^{\prime}\right)$ are finitarily isomorphic if there exists a finitary isomorphism between them. We say following $[54,55]$ that a measurable isomorphism is hyperbolic structure preserving if on sets of full measure it respects the stable and unstable relations (here, the relations of being forwardly/backwardly asymptotic under the shift).

For $S, S^{\prime}$ Markov shifts with given invariant measures $\mu \mapsto \mu^{\prime}$, a magic word isomorphism is a finitary isomorphism $\gamma:(S, \mu) \rightarrow\left(S^{\prime}, \mu^{\prime}\right)$ such that both $\gamma$ and $\gamma^{-1}$ have a magic word. The definition of magic word here is the same as in the 
introduction, with the modifications that conditions (1) and (2) are required to hold only on sets of full measure, and the set of points seeing the magic word $W$ in infinitely many positive and negative coordinates is required to have full measure. The latter condition is automatic for an ergodic measure assigning positive measure to the set $\{x: x[0,|W|-1]=W\}$. (We remark that the definition of magic word isomorphism in [20] is slightly different; a magic word here for $\gamma$ is a magic word in [20] for $\gamma^{-1}$.)

Proposition 3.1. Suppose $\gamma:(S, \mu) \rightarrow\left(S^{\prime}, \mu^{\prime}\right)$ is a magic word isomorphism between Markov shifts endowed with invariant and ergodic probability measures. Then $\gamma$ is a hyperbolic structure preserving isomorphism. If the systems are exponentially recurrent, then $\gamma$ codes exponentially fast.

Proof. With probability one, points will see a magic word infinitely often in positive and in negative coordinates. Therefore, the left tail $x(-\infty, 0]$ of a bisequence $x$ will code some left tail $y(-\infty, m]$ of its image, and likewise for right tails. This proves the a.e. preservation of hyperbolic structure.

Now suppose the systems are exponentially recurrent. Let $V$ be an $S$-word which is a magic word for $\gamma^{-1}$. Without loss of generality, let the index $I$ used in the definition of a magic word in the Introduction be zero. Let $J$ be the length of $V$. For $n>J$, let $E_{n}$ be the set of $S$-points $x$ such that $V$ is not a subword of $x[-n,-1]$, and let $F_{n}$ be the set of $S$-points $x$ such that $V$ is not a subword of $x[1, n]$. Then $x[-n, n]$ codes $(\varphi x)_{0}$ except for a subset of $E_{n} \cup F_{n}$. Let $V$ also denote the set $\{x: x[0, J-1]=V\}$. We have

$$
F_{n}=\left\{x: x \notin \cup_{k=1}^{n-J} S^{-k}(V)\right\} \quad, \quad E_{n}=\left\{x: x \notin \cup_{k=1}^{n-J} S^{k}\left(S^{J} V\right)\right\}
$$

and it follows from Remark 2.2 that

$$
\varlimsup_{n}\left(\mu\left(E_{n} \cup F_{n}\right)\right)^{1 / n} \leq \varlimsup_{n}\left(\mu E_{n}+\mu F_{n}\right)^{1 / n}<1 .
$$

Definitions 3.2. By a measurable system we will mean a measurable map $T: X \rightarrow$ $X$. In this paper, the underlying $\sigma$-algebra will be the Borel $\sigma$-algebra naturally associated to $X$, and the entropy $h(T)$ is defined as the supremum of the measure theoretic entropies with respect to $T$-invariant Borel probabilities. For a measurable system $T: X \rightarrow X$, a subset $N \subset X$ is called entropy-negligible [5] if there is $h<h(T)$ such that $\mu(N)=0$ for every ergodic invariant probability measure $\mu$ with $h(T, \mu)>h$.

Definition 3.3. Two measurable systems $T: X \rightarrow X$ and $S: Y \rightarrow Y$ are entropyconjugate if there exist entropy-negligible subsets $X_{0} \subset X$ and $Y_{0} \subset Y$ and a bimeasurable bijection $\gamma: X \backslash X_{0} \rightarrow Y \backslash Y_{0}$ such that $S \gamma=\gamma T$ for all $x \in X \backslash X_{0}$. Such a map $\gamma$ is called an entropy-conjugacy.

Proposition 3.4. Suppose $S$ and $T$ are almost isomorphic Markov shifts. Then $h(S)=h(T)$, and there are Borel subsets $K$ and $K^{\prime}$ of $S$ and $T$, and a shiftcommuting Borel-measurable bijection $\gamma: K \rightarrow K^{\prime}$, such that the following hold.

(1) $K$ and $K^{\prime}$ are residual subsets of $S$ and $T$ (contain dense $G_{\delta}$ sets).

(2) The map $\gamma$ induces a bijection $\mathcal{M}(K) \rightarrow \mathcal{M}\left(K^{\prime}\right)\left(\mu \mapsto \mu^{\prime}\right.$, say) such that for each such pair $\mu, \mu^{\prime}$ the map $\gamma$ induces an isomorphism $\gamma:(S, \mu) \rightarrow\left(T, \mu^{\prime}\right)$, which is a magic word isomorphism when $\mu$ and $\mu^{\prime}$ have full support. 
(3) $\mathcal{M}(K)$ and $\mathcal{M}\left(K^{\prime}\right)$ contain all ergodic shift-invariant Borel probabilities on $S$ and $T$ with full support, and these correspond under $\gamma$.

(4) If $S$ is $S P R$, then so is $T$, and $\gamma$ is an entropy-conjugacy from $S$ to $T$.

Proof: Suppose $S$ and $T$ are almost isomorphic Markov shifts, i.e., suppose we have another Markov shift $R$ and injective one-block codes $\varphi: R \rightarrow S$ and $\psi: R \rightarrow$ $T$, with magic words $W$ and $W^{\prime}$ respectively. We assume for simplicity that $I=0$ in their definition.

Let $W_{*}$, resp. $W_{*}^{\prime}$, be some $R$-word projecting to $W$, resp. $W^{\prime}$. Pick some other $R$-words $C_{*}, D_{*}, E_{*}$ such that $w_{*}=W_{*} C_{*} W_{*}^{\prime} D_{*} W_{*}^{\prime} E_{*} W_{*}$ is also an $R$-word. Let $w$ be the $S$-word below this $R$-word. Each $x \in \operatorname{Fix}\left(S^{n}\right)$ such that $x[0,|w|-1]=w$ has a unique preimage $\varphi^{-1}(x) \in \operatorname{Fix}\left(R^{n}\right)$ and it satisfies $\varphi^{-1}(x)\left[0,|w|-\left|W_{*}\right|-1\right]=$ $W_{*} C_{*} W_{*}^{\prime} D_{*} W_{*}^{\prime} E_{*}$. Therefore $\psi \varphi^{-1}(x)\left[\left|W_{*} C_{*}\right|,\left|W_{*} C_{*} W_{*}^{\prime} D_{*} W_{*}^{\prime}\right|-1\right]=W^{\prime} C W^{\prime}$ for some $T$-word $C . \psi \varphi^{-1}(x) \in \operatorname{Fix}\left(T^{n}\right)$. Moreover the map $\psi$ is injective on $\operatorname{Fix}\left(R^{n}\right) \cap\left[w_{*}\right]$ as $w_{*}$ projects in $T$ to a magic word.

Thus the number of $n$-periodic sequences in $S$ which starts with $w$ is a lower bound for the number of $n$-periodic sequences in $T$ which starts with $W^{\prime} C W^{\prime}$. The growth rates of these numbers are the entropies. Thus $h(S) \leq h(T)$. By symmetry, $h(S)=h(T)$.

(1) Let $K$ be the image of $\varphi$ in $S$ and let $K^{\prime}$ be the image of $\psi$ in $T$. Let $\gamma: K \rightarrow K^{\prime}$ be the bijection $\gamma=\psi \varphi^{-1}$. For any $n$, the set $K_{n}(W)$ of points in $S$ which see $W$ in at least $n$ negative coordinates and $n$ positive coordinates is a dense open set, and $K$ contains the intersection $K(W)$ of the $K_{n}(W)$. Thus $K$ is a residual subset in $S$, as is $K^{\prime}$ in $T$.

(2) With respect to the measure 1 sets $K(W) \cap \gamma^{-1} K\left(W^{\prime}\right)$ and $\gamma K(W) \cap K\left(W^{\prime}\right)$, the map $\gamma$ is a magic word isomorphism between corresponding measures with full support.

(3) Let $C_{W}=\{x: x[0,|W|-1]=W\}$. Let $\mu$ be ergodic with full support; then $\mu C_{W}>0$, and by ergodicity $\mu(K(W))=1$, so $\mu(K)=1$ and $\mu \in \mathcal{M}(K)$. Suppose $U$ is an open set in $S$; then $U \cap K$ is open in $K$ and $\mu U=\mu(K \cap U)=\mu^{\prime}(\gamma(K \cap U))$. Modulo null sets, non-empty open sets of $K^{\prime}$ contain such non-empty sets $\gamma(K \cap U)$. It follows that $\mu^{\prime}=\gamma \mu$ is a fully supported measure when $\mu$ is. The converse is shown similarly.

(4) $\gamma$ takes the measure of maximal entropy $\mu$ of $S$ (which has full support [21]) to a measure $\mu^{\prime}$ of $T$ which is isomorphic and therefore of maximal entropy (recall $h(S)=h(T)$ ). Because $S$ is SPR, by Proposition $2.3(S, \mu)$ is exponentially recurrent. By [44], exponential recurrence is invariant under finitary isomorphism, so $\mu^{\prime}$ is an exponentially recurrent measure of maximal entropy for $T$. We conclude from Proposition 2.3 that $T$ is SPR.

By Proposition 2.3, the entropies of measures such that $\mu\left(C_{W}\right)=0$ are bounded away from $h(S)$, hence $\mathcal{M}(K)$ contains all ergodic and invariant measures with entropy close to $h(S)$. The same holds for $\mathcal{M}\left(K^{\prime}\right)$. By $(2), \gamma$ is an entropyconjugacy.

Remark 3.5. Suppose $S, S^{\prime}$ are SPR Markov shifts; $\mu, \mu^{\prime}$ are ergodic measures with full support; and $\gamma:(S, \mu) \rightarrow\left(S, \mu^{\prime}\right)$ is a magic word isomorphism. The previous proof shows $\gamma$ must be the restriction of an entropy-conjugacy between $S$ and $S^{\prime}$. 
Remark 3.6. For positive recurrent shifts which are not SPR, the work of Ulf Fiebig [18] shows that recurrent rate invariants of finitary isomorphism are much finer and richer. These also give invariants of almost isomorphism.

Proposition 3.7. The following are true.

(1) Suppose $R \rightarrow S$ and $S \rightarrow T$ are injective one-block codes between Markov shifts, each of which has a magic word. Then their composition is an injective one-block code with a magic word.

(2) Suppose $\sigma_{f}$ is the loop system built from first-return loops to a vertex for a Markov shift $S_{A}$. Then the natural inclusion $\sigma_{f} \rightarrow S_{A}$ is a one-block code with a magic word.

(3) If $R \rightarrow S, R \rightarrow T$ is an almost isomorphism between $S$ and $T$, and $\sigma_{f}$ is a first-return-to-a-vertex loop system for $R$, then there is also an almost isomorphism $\sigma_{f} \rightarrow S, \sigma_{f} \rightarrow T$.

(4) Almost isomorphism defines an equivalence relation on Markov shifts.

Proof. We leave the proof of Proposition 3.7 as an exercise. For the last claim (which we do not use in our proofs), the nontrivial step is to show, given Markov shifts $R_{1}, R_{2}, T$ and injective one block codes with magic words $\varphi: R_{1} \rightarrow T, \varphi_{2}$ : $R_{2} \rightarrow T$, that there is a Markov shift $S$ and injective one block codes $p_{1}: S \rightarrow R_{1}$, $p_{2}: S \rightarrow R_{2}$. For this one can use a standard fiber product construction [31, 35], as follows. Let $S^{\prime}$ be the subset of $R_{1} \times R_{2}$ which consists of points $(x, y)$ such that $\varphi_{1} x=\varphi_{2} y$, and define $p_{1}:(x, y) \mapsto x$ and $p_{2}:(x, y) \mapsto y$. Let $S$ be the unique maximal irreducible Markov shift contained in $S^{\prime}$ which has dense image in $R_{1}$ and in $R_{2}$. It can be verified that $S, p_{1}, p_{2}$ meet the requirements.

Within a class of almost isomorphic Markov shifts, one can freely localize to loop shifts and still capture all ergodic phenomena with full support. However, if a Markov shift $S$ is not SPR, the localization may miss many periodic points and measures of large entropy, as the next example shows.

Example 3.8. Suppose $\sigma_{f}$ is not SPR. Then [45] there is a loop $\ell$ of $\sigma_{f}$ such that the subsystem $T$ which misses $\ell$ has $h(T)=h(S)=\log \lambda$. Pick a vertex on $\ell$ (other than the base vertex for $\sigma_{f}$ ) and let $\sigma_{g}$ be the loop shift based at this vertex. We have the obvious injective block code $\sigma_{g} \rightarrow \sigma_{f}$ with a magic word. However, the supremum of the entropies of ergodic measures assigning measure one to the complement of the image is $\log \lambda$. Therefore, the inclusion $\sigma_{g} \rightarrow \sigma_{f}$ is not an entropy-conjugacy.

\section{ZETA FUnCTIONS}

Suppose $T$ is a bijection of a set, $T: X \rightarrow X$, such that for all $n>0$ the cardinality of the fixed point set of $T^{n}$ (i.e., $\left.\left|\operatorname{Fix}\left(T^{n}\right)\right|\right)$ is finite. Then the ArtinMazur zeta function of $T$ is defined as

$$
\zeta_{T}(z):=\exp \sum_{n \geq 1}\left|\operatorname{Fix}\left(T^{n}\right)\right| \frac{z^{n}}{n} .
$$

Let $\mathcal{O}_{n}(T)$ denote the set of orbits of cardinality $n$ of $T$. We have the well known product formula

$$
\zeta_{T}(z)=\left(\prod_{n=1}^{\infty}\left(1-z^{n}\right)^{\left|\mathcal{O}_{n}(T)\right|}\right)^{-1}
$$


This formula is easily verified when $T$ contains just one finite orbit, and then holds at the level of formal power series by taking countable products. (Note, the zeta function and product formula make perfectly good sense at the level of formal power series even when $\overline{\lim }\left|\operatorname{Fix}\left(T^{n}\right)\right|^{1 / n}=\infty$.) For a loop shift $\sigma_{f}$, with $f(z)=\sum_{n=1}^{\infty} f_{n} z^{n}$, we have

$$
\zeta_{\sigma_{f}}(z)=\frac{1}{1-f(z)} .
$$

We do not know the earliest reference for (4.2); it appears in [57] and in the case $f(z)$ is a polynomial it is a special case of a result proved in the "bigamy" paper [2]. For a detailed proof of a generalization of (4.2), see [24, Theorem 8.2]. We remark, (4.2) in general follows from the case that $f(z)$ is a polynomial, because coefficients of degree at most $n$ in the series on either side of (4.2) match what would be computed for the loop shift for the truncated series $f_{1} z+f_{2} z^{2}+\cdots+f_{n} z^{n}$.

A Markov shift $T$ may fail to have a well defined zeta function in that $\left|\operatorname{Fix}\left(T^{n}\right)\right|$ may be infinite for some or all $n$. To avoid this problem at least when the entropy is finite, we use, as in $[24,49]$, a local zeta function, i.e., the zeta function of the loop shift given by the return loops to a given vertex $s$. For a finite entropy Markov shift, for every $n$ there are only finitely many first return loops to $s$ of length $n$, so the local zeta function must be well defined.

Proposition 4.3. Let $\sigma_{f}$ be a mixing SPR loop shift with finite positive entropy $\log (\lambda)$. Let $\zeta(z)$ denote its zeta function $\zeta_{\sigma_{f}}(z)$. Then $\zeta(z)$ is holomorphic in $|z|<1 / \lambda$ and has a meromorphic extension to a larger disk (all our disks are centered at zero). More precisely, $(1-\lambda z) \zeta(z)$ is holomorphic and nonzero in some disk $|z|<r$ with $r>1 / \lambda$.

Proposition 4.3 is in the treatise of Gurevich and Savchenko [24, Proposition 9.2]. We give a proof for completeness.

PROOF: By definition of SPR, $f$ is holomorphic on a disk of radius strictly greater than $\lambda^{-1}$. Its power series has no constant term, hence it is not constantly equal to 1. It then follows from (4.2) that $\zeta(z)$ has a meromorphic extension to this disk with poles exactly at points $z$ where $f(z)=1$. Let $\zeta(z)$ also denote this meromorphic extension.

Now $f\left(\lambda^{-1}\right)=1$ as $\sigma_{f}$ is not transient. Hence $\lambda^{-1}$ is a pole of $\zeta(z)$. As $f(z)$ is a power series with non-negative coefficients not all zero, $f^{\prime}\left(\lambda^{-1}\right)>0$. In particular, $f^{\prime}\left(\lambda^{-1}\right)$ is non-zero and the pole at $\lambda^{-1}$ is, as claimed, a simple pole.

The mixing assumption implies that there are no other poles on the circle $|z|=$ $\lambda^{-1}$. Indeed, let $|z|=\lambda^{-1}$. We have

$$
\left|\sum_{n \geq 1} f_{n} z^{n}\right| \leq \sum_{n \geq 1} f_{n}\left|z^{n}\right|=1
$$

and the inequality is an equality iff $z^{n}=\left|z^{n}\right|=\lambda^{-n}$ for all $n \geq 1$ such that $f_{n} \neq 0$. Such a $z$ must be $\omega \lambda^{-1}$ with $\omega$ a root of unity of some order $q \geq 1$ such that $f_{n} \neq 0 \Longrightarrow q \mid n$. Therefore $q$ divides the period of $\sigma_{f}$. But $\sigma_{f}$ is mixing and therefore this period is 1 . In particular, $q=1$ and $z=\lambda^{-1}$. By compactness, $z=\lambda^{-1}$ is the only pole of $\zeta(z)$ on some disk of radius $>\lambda^{-1}$.

The following corollary will be essential to the proof of our main result (6.4). 
Corollary 4.4. Let $\sigma_{f}$ and $\sigma_{g}$ be mixing SPR loop shifts of equal finite entropy $\log (\lambda)>0$. Then

$$
\varlimsup_{n}|| \mathcal{O}_{n}\left(\sigma_{f}\right)|-| \mathcal{O}_{n}\left(\sigma_{g}\right)||^{1 / n}<\lambda .
$$

Proof: Set $a_{n}=\left|\operatorname{Fix}\left(\left(\sigma_{f}\right)^{n}\right)\right|-\left|\operatorname{Fix}\left(\left(\sigma_{g}\right)^{n}\right)\right|$ and $b_{n}=\left|\mathcal{O}_{n}\left(\sigma_{f}\right)\right|-\left|\mathcal{O}_{n}\left(\sigma_{g}\right)\right|$. Without loss of generality, we assume $b_{n} \neq 0$ for infinitely many $n$, so $\overline{\lim }\left|b_{n}\right|^{1 / n} \geq 1$ and $\varlimsup\left|a_{n}\right|^{1 / n} \geq 1$. Because $a_{n}=\sum_{k \mid n} k b_{k}$, and by Möbius inversion $n b_{n}=$ $\sum_{k \mid n} \mu(n / k) a_{k}$, it then follows that $\varlimsup\left|b_{n}\right|^{1 / n}=\varlimsup\left|a_{n}\right|^{1 / n}$.

By Proposition 4.3,

$$
\frac{\zeta_{\sigma_{f}}(z)}{\zeta_{\sigma_{g}}(z)}=\exp \sum_{n \geq 1}\left(\left|\operatorname{Fix}\left(\left(\sigma_{f}\right)^{n}\right)\right|-\left|\operatorname{Fix}\left(\left(\sigma_{g}\right)^{n}\right)\right|\right) \frac{z^{n}}{n}
$$

is a holomorphic function on a disk of radius $r>1 / \lambda$, and consequently so is $\sum\left(a_{n} / n\right) z^{n}$. Therefore $\varlimsup\left|a_{n}\right|^{1 / n}<\lambda$, which implies $\varlimsup\left|b_{n}\right|^{1 / n}<\lambda$.

\section{The Loops Lemma}

Given a power series $k$ with zero constant term, we use the standard notation $k^{*}$ to denote the power series $1+k+k^{2}+\cdots=1 /(1-k)$. Similarly, given a set $K$ of words, containing only finitely many words of any fixed length, we let $K^{*}$ denote the set of words which are concatenations of words in $K$, and we include in $K^{*}$ the empty word, which we regard as an identity element for concatenation.

The construction of the next lemma is lifted from [20].

Lemma 5.1. Suppose $f=h+k$ where $h$ and $k$ are formal power series in $z \mathbb{Z}_{+}[[z]]$ which are not identically zero. Then $1-h k^{*}=(1-f) /(1-k)$, and there is an injective one-block code $\psi$ from $\sigma_{h k^{*}}$ to $\sigma_{f}$ which has a magic word. If additionally $h\left(\sigma_{k}\right)<h\left(\sigma_{f}\right)$, then $\psi$ is also an entropy-conjugacy.

PROOF: The equality is an application of the geometric series.

We have $h=\sum_{n=1}^{\infty} h_{n} z^{n}$ and $k=\sum_{n=1}^{\infty} k_{n} z^{n}$. Let $\mathcal{G}_{f}$ be a loop graph for $f=h+k$, with $\mathcal{L}$ the set of (first return) loops of $\mathcal{G}$. Choose subsets $\mathcal{H}$ and $\mathcal{K}$ of $\mathcal{L}$ such that $\mathcal{L}$ is the disjoint union of $\mathcal{H}$ and $\mathcal{K}$; and for every $n$, the number of loops of length $n$ in $\mathcal{H}$ is $h_{n}$; and for every $n$, the number of loops of length $n$ in $\mathcal{K}$ is $k_{n}$. Let $\mathcal{L}^{\prime}$ be the loop set of a loop graph $\mathcal{G}_{h k^{*}}$ for the series $h k^{*}=h\left(1+k+k^{2}+\cdots\right)$. Choose a bijection $\beta: \mathcal{L}^{\prime} \rightarrow \mathcal{H} \mathcal{K}^{*}$ respecting word length and let $\beta$ define a labeling of $\mathcal{L}^{\prime}$, and thereby a one-block code $\psi$ from $\sigma_{h k^{*}}$ to $\sigma_{f}$. Each loop $l$ of $\mathcal{L}^{\prime}$ has a label $\widehat{l}$ which has a nonempty prefix which is an element of $\mathcal{H}$, and no word of $\mathcal{H}$ occurs in $\widehat{l}$ except as a prefix. Consequently any word of $\mathcal{H}$ is a magic word for $\psi$.

It follows in particular that $\psi$ is an isomorphism w.r.t. any ergodic and invariant measure, unless the measure avoids all words of $\mathcal{H}$ and thus lives on $\sigma_{k}$. The entropy of such a measure is bounded by $h\left(\sigma_{k}\right)$, so $\varphi$ is an entropy-conjugacy if $h\left(\sigma_{k}\right)<h\left(\sigma_{f}\right)$.

The rest of this section is devoted to the following lemma, which records the key features of our main construction.

Lemma 5.2 (Loops Lemma). Let $\sigma_{f}$ be a mixing loop shift, $f=\sum_{n=1}^{\infty} f_{n} z^{n}$, with $0<h\left(\sigma_{f}\right)=\log (\lambda) \leq \infty$. Let $\left(r_{k}\right)_{k=1}^{\infty}$ be a nondecreasing sequence of positive 
integers $^{1}$. Let $R_{n}=\#\left\{k: r_{k}=n\right\}$. Set $f^{<1>}=f$. Given $f^{<k>}$, define the series $f^{<k+1>}=\sum_{n=1}^{\infty} f_{n}^{<k+1>} z^{n}$ by the equation

$$
f^{<k+1>}(z)=\left(f^{<k>}(z)-z^{r_{k}}\right) \sum_{n=0}^{\infty} z^{n r_{k}}
$$

or equivalently

$$
1-f^{<k+1>}(z)=\frac{1-f^{<k>}(z)}{\left(1-z^{r_{k}}\right)} .
$$

Assume the pair $\left(f,\left(r_{k}\right)_{k=1}^{\infty}\right)$ satisfies the positivity condition

$$
f_{r_{k}}^{<k>} \geq 1, \quad k \in \mathbb{N}
$$

(which guarantees every $\left.f^{<k>}(z) \in z \mathbb{Z}_{+}[[z]]\right)$. Because $\lim _{k} r_{k}=\infty$, it follows that for every $n$ in $\mathbb{N}$, the sequence $\left(f_{n}^{<k>}\right)_{k=1}^{\infty}$ is eventually constant. Define $f_{n}^{<\infty>}=$ $\lim _{k} f_{n}^{<k>}$ and $f^{<\infty>}=\sum_{n=1}^{\infty} f_{n}^{<\infty>} z^{n}$. Let $f^{(n)}$ be the series $f^{<k>}$ where $k$ is defined by the condition $r_{j}<n$ if and only if $j<k$.

Then there is a one-block code $\varphi: \sigma_{f<\infty>} \rightarrow \sigma_{f}$ with the following properties.

(1) $\varphi$ is injective.

(2) For every $n, 1-f^{(n+1)}(z)=\left(1-f^{(n)}(z)\right) /\left(\left(1-z^{n}\right)^{R_{n}}\right)$.

(3) $1-f^{<\infty>}(z)=(1-f(z)) /\left(\prod_{n=1}^{\infty}\left(1-z^{n}\right)^{R_{n}}\right)$.

(4) If $\varlimsup_{\lim _{n}}\left(R_{n}\right)^{1 / n}<\lambda$, then $h\left(\sigma_{f<\infty>}\right)=\log (\lambda)$.

(5) If $\varlimsup_{n}\left(R_{n}\right)^{1 / n}<\lambda$, then $\sigma_{f}$ is SPR if and only if $\sigma_{f<\infty>}$ is $S P R$.

(6) Suppose

$$
R_{n} \leq f_{n} \text { for all } n \text {, and also } R_{n}<f_{n} \text { for } n=r_{1} .
$$

Then the map $\varphi$ can be chosen to have a magic word.

(7) Suppose in addition to (5.4) that $\overline{\lim }_{n}\left(R_{n}\right)^{1 / n}<\lambda$. Then the map $\varphi$ can be chosen to have a magic word and also to be an entropy-conjugacy.

PROOF: Construction of $\widehat{\mathcal{G}}^{<k>}$. Inductively, we will define a sequence of labeled graphs $\widehat{\mathcal{G}}^{<k>}$, such that the underlying graph $\mathcal{G}^{<k>}$ is a loop graph for $f^{<k>}$, the labeling set is the edge set of $\mathcal{G}^{<1>}$, and the labeled graph $\widehat{\mathcal{G}}^{<k>}$ defines a one-block code $\varphi_{k}: \sigma_{f<k>} \rightarrow \sigma_{f}$ (here, $\left(\varphi_{k} y\right)_{i}$ is the label of the edge $\left.y_{i}\right)$. We let $\mathcal{L}^{<k>}$ denote the set of (first return) loops of $\mathcal{G}^{<k>}$, and we let $\widehat{\mathcal{L}}^{<k>}$ denote the labeled loops, from $\widehat{\mathcal{G}}^{<k>}$.

If $l$ is a path $l_{1} \cdots l_{j}$ of $j$ edges in a labeled graph, then we denote by $\widehat{l}$ its label, which is a word $\widehat{l}_{1} \cdots \widehat{l}_{j}$, where $\widehat{l}_{i}$ is the label of $l_{i}$. Similarly when we label a path $l_{1} \cdots l_{j}$ with a word $w_{1} \cdots w_{j}$, we are defining $\widehat{l}_{i}=w_{i}$.

Choose $\mathcal{G}^{<1>}$ to be a loop graph for $f=f^{<1>}$. Define $\widehat{\mathcal{G}}^{<1>}$ by giving each edge a distinct label. Given $\widehat{\mathcal{G}}^{<k>}$, we construct $\widehat{\mathcal{G}}^{<k+1>}$ from $\widehat{\mathcal{G}}^{<k>}$ as follows. ( $l^{0}$ will denote the empty word, so $c l^{0}=c$.)

- Choose a loop $l^{<k>}=l$ of length $r_{k}$ in $\mathcal{G}^{<k>}$ (at least one such loop exists by the positivity condition (5.3)).

- Now $\widehat{\mathcal{G}}^{<k+1>}$ is the labeled loop graph with exactly the following set of labeled loops: for every loop $c$ in $\mathcal{L}^{<k>} \backslash\{l\}$, for $n=0,1,2,3, \ldots$, put in $\mathcal{G}^{<k+1>}$ one loop $\ell(c, n)$, of length $|c|+n r_{k}$, with label $\widehat{c l^{n}}$.

\footnotetext{
${ }^{1} r_{k}$ will be the length of the orbit to be deleted at stage $k$.
} 
The rule $\ell(c, n) \mapsto c l^{n}$ defines a map $\mathcal{L}^{<k+1>} \rightarrow\left(\mathcal{L}^{<k>}\right)^{*}$ which determines a one block code $\iota_{k+1}: \sigma_{f<k+1>} \rightarrow \sigma_{f<k>}$ which respects the labeling. Therefore the map $\varphi_{k+1}$ is the composition of $\iota_{k+1}, \ldots, \iota_{2}$. Clearly $f_{m}^{<k+1>}=f_{m}^{<k>}-1$ if $m=r_{k}$, and $f_{m}^{<k>}=f_{m}^{<k+1>}$ if $m<r_{k}$. Each sequence $\left(f_{n}^{<k>}\right)_{k=1}^{\infty}$ is eventually constant and the positivity condition (5.3) does guarantee each $f^{<k>} \in z \mathbb{Z}_{+}[[z]]$. Moreover, the set of labeled loops in $\widehat{\mathcal{G}}^{<k>}$ of length $n$ or less does not change once $r_{k}>n$. We define $\widehat{\mathcal{G}}^{<\infty>}$ to be the labeled loop graph whose labeled loops of length $n$ are those in all but finitely many of the $\widehat{\mathcal{G}}^{<k>}$. Then $\mathcal{G}^{<\infty>}$ is a loop graph for $f<\infty>$ and the labels of $\widehat{\mathcal{G}}^{<\infty>}$ define a one block code $\varphi: \sigma_{f<\infty>} \rightarrow \sigma_{f}$.

Injectivity. For every $k$ in $\mathbb{N}$, the map $\iota_{k+1}: \sigma_{f<k+1>} \rightarrow \sigma_{f<k>}$ is injective. (The map $\iota_{k+1}$ is not surjective; a point $w$ of $\sigma_{f<k>}$ fails to be in the image of of $\iota_{k+1}$ if and only if $w$ is forwardly or backwardly asymptotic to the periodic orbit $\cdots l^{<k>} l^{<k>} l^{<k>} \ldots$.) It follows for $1 \leq k<\infty$ that $\varphi_{k}: \sigma_{f<k>} \rightarrow \sigma_{f}$ is injective, with $\operatorname{Image}\left(\varphi_{1}\right) \supset \operatorname{Image}\left(\varphi_{2}\right) \supset \operatorname{Image}\left(\varphi_{3}\right) \supset \ldots$. For $x \in \sigma_{f}$ and $1 \leq k \leq \infty$, define $V_{k}(x)$ to be the set of integers $i$ such that there exists $y \in \sigma_{f<k>}$ such that $\varphi_{k}(y)=x$ and the edge $y_{i}$ begins at the base vertex of $\mathcal{G}^{<k>}$. (Here $\varphi_{\infty}=\varphi$.) The injectivity of $\varphi_{k}$ and the structure of $\iota_{k}$ show for any given $x$ that $V_{1}(x) \supset V_{2}(x) \supset V_{3}(x) \cdots$. A point $x$ of $\sigma_{f}$ will have a preimage under $\varphi$ in $\sigma_{f<\infty>}$ if and only if $\cap_{1 \leq k<\infty} V_{k}(x)$ is unbounded above and below, in which case

$$
V_{\infty}(x)=\bigcap_{1 \leq k<\infty} V_{k}(x)
$$

Because $\mathcal{G}^{<\infty>}$ inherits from the $\mathcal{G}^{<k>}$ 's the property that distinct loops have distinct labels, this implies that $\varphi$ is injective.

Zeta function and entropy. For each $n$, if $r_{k}>n$ then the number of loops of length $n$ or less in $\mathcal{G}^{<k>}$ is the same as in $\mathcal{G}^{<\infty>}$. At any finite stage $k$, $\left|\mathcal{O}_{n}\left(\sigma_{f<k+1>}\right)\right|=\left|\mathcal{O}_{n}\left(\sigma_{f<k>}\right)\right|$, except for the $R_{n}$ values of $k$ at which $r_{k}=n$; at each of these values, $\left|\mathcal{O}_{n}\left(\sigma_{f<k+1>}\right)\right|=\left|\mathcal{O}_{n}\left(\sigma_{f<k>}\right)\right|-1$. This description proves (2) and also that $\left|\mathcal{O}_{n}\left(\sigma_{f}<\infty>\right)\right|=\left|\mathcal{O}_{n}\left(\sigma_{f}\right)\right|-R_{n}$, for all $n$. Consequently, using the product formula for the zeta function, we also have

$$
1-f^{<\infty>}=\frac{\prod_{n=1}^{\infty}\left(1-z^{n}\right)^{\left|\mathcal{O}_{n}\left(\sigma_{f}\right)\right|}}{\prod_{n=1}^{\infty}\left(1-z^{n}\right)^{R_{n}}}=\frac{1-f(z)}{\prod_{n=1}^{\infty}\left(1-z^{n}\right)^{R_{n}}}
$$

which proves (3). By the same product formula,

$$
\left(\prod_{n=1}^{\infty}\left(1-z^{n}\right)^{R_{n}}\right)^{-1}=\exp \sum_{n=1}^{\infty} \frac{1}{n} Q_{n} z^{n}:=q(z)
$$

where $Q_{n}=\sum_{k \mid n} k R_{k}$ and therefore $\varlimsup \overline{\lim }\left(Q_{n}\right)^{1 / n}=\varlimsup \lim \left(R_{n}\right)^{1 / n}=1 / \operatorname{rad}(q)$. Finally, given $\overline{\lim }\left(R_{n}\right)^{1 / n}<\lambda$, the claim (4) follows from

$$
\begin{aligned}
h\left(\sigma_{f}\right) & =\varlimsup_{n} \log \left|\mathcal{O}_{n}\left(\sigma_{f}\right)\right|^{1 / n}=\varlimsup_{n} \log \left(\left|\mathcal{O}_{n}\left(\sigma_{f}\right)\right|-R_{n}\right)^{1 / n} \\
& =\varlimsup_{n} \log \left|\mathcal{O}_{n}\left(\sigma_{f<\infty>}\right)\right|^{1 / n}=h\left(\sigma_{f<\infty>}\right) .
\end{aligned}
$$

Strong positive recurrence. We have $\operatorname{rad}\left(f^{<\infty>}\right)=\min \{\operatorname{rad}(f), \operatorname{rad}(q)\}$. If we assume $\varlimsup_{n}\left(R_{n}\right)^{1 / n}<\lambda$, it follows that $\operatorname{rad}(q)<1 / \lambda$ and

$$
\operatorname{rad}\left(f^{<\infty>}\right)>\frac{1}{\lambda} \Longleftrightarrow \operatorname{rad}(f)>\frac{1}{\lambda}
$$


Therefore $\sigma_{f<\infty}>$ is SPR if and only if $\sigma_{f}$ is SPR, proving (5).

A magic word. Consider the inductive construction in terms of the sequence $\left(\widehat{\mathcal{G}}^{<k>}\right), k=1,2, \ldots$. The loops of $\mathcal{G}^{<k+1>}$ are loops $\ell(c, n)$ and the label of $\ell(c, n)$ in $\widehat{\mathcal{G}}^{<k+1>}$ is $\hat{c} l^{n}$.

Assume (5.4) holds. Let $N=r_{1}$, so, the first deleted loop $l^{<1>}$ will have length $N$. Pick a loop $W$ of length $N$. At this initial stage, in $\widehat{\mathcal{G}}^{<1>}$ every loop is labeled by its own name, and symbols of $W$ occur in labels of no loop other than $W$ itself. So, for $\widehat{\mathcal{G}}=\widehat{\mathcal{G}}^{<1>}$, we have the following

Condition 5.6. A symbol of $W$ can occur in a label $\widehat{l}$ of a loop $l$ in $\widehat{\mathcal{G}}$ only as part of an initial segment of $\widehat{l}$ which equals $W$

Now suppose that (5.6) holds for $\widehat{\mathcal{G}}=\widehat{\mathcal{G}}^{<k>}$, and the loop $l^{<k>}$ has the property that no symbol of $W$ occurs in $\widehat{l}^{<k>}$. It then follows from the construction that (5.6) holds for $\widehat{\mathcal{G}}=\widehat{\mathcal{G}}^{<k+1>}$. However, because (5.4) holds, for each $k$ we can pick for $l^{<k>}$ a loop which already exists in $\mathcal{G}^{<1>}$ and which is not $W$, and which therefore has a label using no symbol of $W$.

Inductively, then, we construct the $\widehat{\mathcal{G}}^{<k>}$ to satisfy (5.6), and in the limit obtain $\widehat{\mathcal{G}}^{<\infty>}$. The map $\varphi$ is the one-block code from $\sigma_{f<\infty>}$ to $\sigma_{f}$ defined by the labeling of loops in $\widehat{\mathcal{G}}^{<\infty>}$. We claim that $W$ is a magic word for $\varphi$. To see this, first note that the labeling of loops in $\widehat{\mathcal{G}}^{<\infty>}$ inherits the following properties from the $\widehat{\mathcal{G}}^{<k>}$ :

(1) distinct loops have distinct labels

(2) a symbol of $W$ can occur in a label $\widehat{l}$ of a loop $l$ in $\widehat{\mathcal{G}}^{<\infty>}$ only as part of an initial segment of $\widehat{l}$ which equals $W$

(3) if $W D W$ is a word in $\sigma_{f}$, then $W D$ is the label of a unique concatenation of loops in $\widehat{\mathcal{G}}^{<\infty>}$.

Notice that if (3) did not hold, then $\varphi$ would not be injective, contrary to what we established.

It follows from property (3) and the remark before (5.5) that whenever a point $x$ of $\sigma_{f}$ has the property that the set $\{n: x[n, n+|W|-1]=W\}$ is unbounded above and below, then a preimage of $x$ can be constructed in $\sigma_{f<\infty>}$.

Finally, suppose $x[i, 2|W|+|D|-1]=W D W$ and $\varphi y=x$. It follows from (2) that both of the edges $y[i]$ and $y[i+|W|+|D|-1]$ have as initial vertex the base vertex of the loop graph $\widehat{\mathcal{G}}^{<\infty>}$, and therefore $y[i,|W|+|D|-1]$ is a concatenation of loops in $\widehat{\mathcal{G}}^{<\infty>}$. By (3), this concatenation is unique. Therefore, $W$ is a magic word for $\varphi$, proving $(6)$.

Entropy-conjugacy. We remark that when $S$ and $T$ are SPR, it follows already from (3.5) that the map $\varphi$ provided by (6) is an entropy conjugacy. We now proceed to the general case, assuming (5.4) and $\varlimsup_{n}\left(R_{n}\right)^{1 / n}<\lambda$.

Let $\sigma_{R}$ be the loop shift corresponding to $R(z)=\sum_{n=1}^{\infty} R_{n} z^{n}$. Appealing to (5.4), we regard $\sigma_{R}$ as a subsystem of $\sigma_{f}$. We construct the map $\varphi$ as above, with the following additional constraint: for every $k$, the deleted loop $l^{<k>}$ is chosen to be a loop of $\sigma_{R}$. As in the argument for (6), it follows that any loop $W$ of $\sigma_{f}$ which is not a loop of $\sigma_{R}$ must be a magic word for $\varphi$.

As $\varphi$ is injective, it is a measure-preserving isomorphism $\left(\sigma_{f<\infty>}, \nu\right) \rightarrow\left(\sigma_{f}, \varphi \nu\right)$ for any invariant probability measure $\nu$ of $\sigma_{f<\infty>}$. Let us consider an ergodic and invariant probability measure $\mu$ for $\sigma_{f}$ such that $\varphi^{-1}$ fails to be a.e. defined (otherwise $\varphi:\left(\sigma_{f<\infty>}, \varphi^{-1} \mu\right) \rightarrow\left(\sigma_{f}, \mu\right)$ would be an isomorphism). If $W$ is a loop 
of $\sigma_{f}$ which is not a loop of $\sigma_{R}$, then $\mu\{x: x[0,|W|-1]\}$ must be zero. Therefore $\mu$ is supported on $\sigma_{R}$.

It is enough now to prove $h\left(\sigma_{R}\right)<h\left(\sigma_{f}\right)$. Assume first that $\sigma_{R}$ is not SPR. Then $h\left(\sigma_{R}\right)=\log \varlimsup_{n} R_{n}^{1 / n}<h\left(\sigma_{f}\right)$, proving the claim in this case. Assume now that $\sigma_{R}$ is SPR. Pick some $n_{0}$ such that $R_{n_{0}}<f_{n_{0}}$ and let $R_{n}^{\prime}=R_{n}$ for all $n \neq n_{0}$ and $R_{n_{0}}^{\prime}=R_{n_{0}}+1$. Clearly $\overline{\lim }_{n} R_{n}^{\prime 1 / n}=\overline{\lim }_{n} R_{n}^{1 / n}<h\left(\sigma_{R}\right) \leq h\left(\sigma_{R^{\prime}}\right)$. It follows that $\sigma_{R^{\prime}}$ is SPR, hence by (1) of Proposition 2.3, $h\left(\sigma_{R}\right)<h\left(\sigma_{R^{\prime}}\right) \leq h\left(\sigma_{f}\right)$. This implies the claim.

\section{Main Results}

Lemma 6.1. Suppose $\sigma_{F}$ and $\sigma_{G}$ are mixing loop shifts of equal entropy $\log (\lambda)>0$, and $1 \leq \beta<\lambda$. Then for any sufficiently large $N \in \mathbb{N}$, there are loop shifts $\sigma_{f}$ and $\sigma_{g}$ such that

(1) There are injective one-block codes $\sigma_{f} \rightarrow \sigma_{F}$ and $\sigma_{g} \rightarrow \sigma_{G}$, each of which is an entropy-conjugacy with a magic word.

(2) $\left|\mathcal{O}_{n}\left(\sigma_{f}\right)\right|=\left|\mathcal{O}_{n}\left(\sigma_{g}\right)\right|=0$ for $n<N$,

(3) $\left|\mathcal{O}_{n}\left(\sigma_{f}\right)\right|-\left|\mathcal{O}_{n}\left(\sigma_{g}\right)\right|=\left|\mathcal{O}_{n}\left(\sigma_{F}\right)\right|-\left|\mathcal{O}_{n}\left(\sigma_{G}\right)\right|, \quad n \geq N$, and

(4) $\min \left\{f_{n}, g_{n}\right\} \geq \beta^{n}, \quad n \geq N$.

Proof: Because $\beta<\lambda$ and

$$
\lim _{n}\left|\mathcal{O}_{n}\left(\sigma_{F}\right)\right|^{1 / n}=\lambda=\lim _{n}\left|\mathcal{O}_{n}\left(\sigma_{G}\right)\right|^{1 / n}
$$

for $N$ sufficiently large we may assume

$$
n \geq N \Longrightarrow \min _{n}\left\{\left|\mathcal{O}_{n}\left(\sigma_{F}\right)\right|,\left|\mathcal{O}_{n}\left(\sigma_{G}\right)\right|\right\} \geq 2\left\lceil\beta^{n}\right\rceil
$$

where $\left\lceil\beta^{n}\right\rceil$ denotes the integer ceiling of $\beta^{n}$. Fix such an $N$. Beginning with $f=F$, we apply Lemma 5.1 repeatedly with $k=z^{n}$, where $n$ is the length of a shortest loop, until all loops of length less than $N$ have been deleted. This produces a series

$$
\bar{F}=\sum_{n=N}^{\infty} \bar{F}_{n} z^{n}
$$

such that $\sigma_{\bar{F}} \rightarrow \sigma_{F}$ is an injective one-block code which has a magic word and is an entropy-conjugacy (indeed, $h\left(\sigma_{z^{n}}\right)=0$ ). We also have:

$$
\begin{aligned}
1-\bar{F} & =(1-F) /\left(\prod_{1 \leq n<N}\left(1-z^{n}\right)^{\left|\mathcal{O}_{n}\left(\sigma_{F}\right)\right|}\right) \\
& =(1-F) \prod_{1 \leq n<N}\left(\sum_{k=0}^{\infty} z^{n k}\right)^{\left|\mathcal{O}_{n}\left(\sigma_{F}\right)\right|} .
\end{aligned}
$$

Now $\left|\mathcal{O}_{n}\left(\sigma_{\bar{F}}\right)\right|=\left|\mathcal{O}_{n}\left(\sigma_{F}\right)\right| \geq 2\left\lceil\beta^{n}\right\rceil$ for $n \geq N$, and $\bar{F}_{n}=\left|\mathcal{O}_{n}\left(\sigma_{\bar{F}}\right)\right|$ for $N \leq n<$ $2 N$. Let $b_{n}=\left\lceil\beta^{n}\right\rceil$ and define the series (polynomial)

$$
b(z)=\sum_{n=N}^{2 N-1} b_{n} z^{n}
$$

(so, $\bar{F}_{n}-b_{n}>\beta^{n}$ for $\left.N \leq n<2 N\right)$. Set $h=\bar{F}-b$ and define $f=h b^{*}=$ $h\left(1+b+b^{2}+\cdots\right)$. By Lemma 5.1, there is an injective one-block code $\sigma_{f} \rightarrow \sigma_{\bar{F}}$ which has a magic word and is an entropy-conjugacy. Indeed, $h\left(\sigma_{b}\right)<h\left(\sigma_{F}\right)=h\left(\sigma_{f}\right)$ as 
$b$ defines a finite state Markov shift whose entropy must increase when adding any of the remaining loops of $\sigma_{F}$. Also, the choice of $b$ implies $f_{n} \geq \beta^{n}$ for $n \geq N$.

Similarly construct $\bar{G}$ from $G$, and for $b(z)$ exactly as above set $k=\bar{G}-b$ and $g=k b^{*}$. We have proven (1), (2) and (4). To prove (3), we compute

$$
\begin{aligned}
\left|\mathcal{O}_{n}\left(\sigma_{f}\right)\right|-\left|\mathcal{O}_{n}\left(\sigma_{g}\right)\right| & =\left(\left|\mathcal{O}_{n}\left(\sigma_{\bar{F}}\right)\right|-\left|\mathcal{O}_{n}\left(\sigma_{b}\right)\right|\right)-\left(\left|\mathcal{O}_{n}\left(\sigma_{\bar{G}}\right)\right|-\left|\mathcal{O}_{n}\left(\sigma_{b}\right)\right|\right) \\
& =\left|\mathcal{O}_{n}\left(\sigma_{\bar{F}}\right)\right|-\left|\mathcal{O}_{n}\left(\sigma_{\bar{G}}\right)\right| \\
& =\left|\mathcal{O}_{n}\left(\sigma_{F}\right)\right|-\left|\mathcal{O}_{n}\left(\sigma_{G}\right)\right|, \quad n \geq N
\end{aligned}
$$

Theorem 6.2. Suppose $\sigma_{F}$ and $\sigma_{G}$ are loop shifts of equal period and equal entropy $\log (\lambda)$ (possibly infinite), and

$$
\left.\varlimsup_{n}|| \mathcal{O}_{n}\left(\sigma_{F}\right)|-| \mathcal{O}_{n}\left(\sigma_{G}\right)\right|^{1 / n}<\lambda .
$$

Then $\sigma_{F}$ and $\sigma_{G}$ are almost isomorphic, by an almost isomorphism which induces an entropy-conjugacy of $\sigma_{F}$ and $\sigma_{G}$.

Proof: Without loss of generality, suppose $\lambda>1$. To begin, suppose the period is one, i.e. $\sigma_{F}$ and $\sigma_{G}$ are mixing. Let $\gamma=\varlimsup\left|\mathcal{l i m}_{n}\left(\sigma_{F}\right)\right|-\left|\mathcal{O}_{n}\left(\sigma_{G}\right)\right|^{1 / n}$. Choose $\beta$ such that $\gamma<\beta<\lambda$. Choose $f, g$ and $N$ to satisfy the statement of Lemma 6.1 for these $\beta, \lambda, F$ and $G$, with $N$ large enough that

$$
n \geq N \Longrightarrow|| \mathcal{O}_{n}\left(\sigma_{F}\right)|-| \mathcal{O}_{n}\left(\sigma_{G}\right)|| \leq \beta^{n}-1 .
$$

It suffices now to prove the theorem with $\sigma_{f}$ and $\sigma_{g}$ in place of $\sigma_{F}$ and $\sigma_{G}$.

We will apply the Loops Lemma 5.2 to $\sigma_{f}$ and to $\sigma_{g}$, using sequences $\left(R_{n}\right)$ defined for $f$ and for $g$ as follows:

$$
\begin{aligned}
& R_{n}(f)=\max \left\{0,\left|\mathcal{O}_{n}\left(\sigma_{f}\right)\right|-\left|\mathcal{O}_{n}\left(\sigma_{g}\right)\right|\right\}, \\
& R_{n}(g)=\max \left\{0,\left|\mathcal{O}_{n}\left(\sigma_{g}\right)\right|-\left|\mathcal{O}_{n}\left(\sigma_{f}\right)\right|\right\} .
\end{aligned}
$$

These sequences $\left(R_{n}\right)$ determine the corresponding sequences $\left(r_{k}\right)$ of the Loops Lemma, as well as Loops Lemma sequences of functions $f^{(n)}$ and $g^{(n)}$, the corresponding graphs $\mathcal{G}^{(n)}(f)$ and $\mathcal{G}^{(n)}(g)$, and the limit functions $f^{\langle\infty\rangle}$ and $g^{\langle\infty\rangle}$. Recall $f_{n}^{(n)}$ and $g_{n}^{(n)}$ denote the number of (first return) loops of length $n$ in $\mathcal{G}^{(n)}(f)$ and $\mathcal{G}^{(n)}(g)$, respectively. Using (2) of the Loops Lemma, it is easy to verify by induction that for every $n$,

- for $k<n$, the number of loops of length $k$ in $\mathcal{G}^{(n)}(f)$ and $\mathcal{G}^{(n)}(g)$ is the same, and

- $f_{n}^{(n)}-g_{n}^{(n)}=\left|\mathcal{O}_{n}\left(\sigma_{f}\right)\right|-\left|\mathcal{O}_{n}\left(\sigma_{g}\right)\right|$.

It follows that $f^{\langle\infty\rangle}=g^{\langle\infty\rangle}$. Consequently, there is a map $\sigma_{f}\langle\infty\rangle \rightarrow \sigma_{g}\langle\infty\rangle$ which is a one-block code renaming edges, with inverse likewise a one-block code (here all nonempty words are magic words). By symmetry, it now remains to show that the injective one-block code $\sigma_{f}<\infty>\rightarrow \sigma_{f}$ of the Loops Lemma can be chosen to have a magic word and to be an entropy-conjugacy.

Applying the definition of $R_{n}(f)$, Lemma 6.1(3), the bound (6.3), and Lemma $6.1(4)$, we have for $n \geq N$ that

$$
\begin{aligned}
R_{n}(f) & =\max \left\{0,\left|\mathcal{O}_{n}\left(\sigma_{f}\right)\right|-\left|\mathcal{O}_{n}\left(\sigma_{g}\right)\right|\right\}=\max \left\{0,\left|\mathcal{O}_{n}\left(\sigma_{F}\right)\right|-\left|\mathcal{O}_{n}\left(\sigma_{G}\right)\right|\right\} \\
& \leq \beta^{n}-1 \leq f_{n}-1 .
\end{aligned}
$$


Moreover, by Lemma 6.1(2), the length $r_{1}$ of the first loop of $\sigma_{f}$ deleted in the Loops Lemma construction is equal to $N$. Therefore conditions (6) and (7) of the Loops Lemma are satisfied, and $\sigma_{f<\infty} \rightarrow \sigma_{f}$ can be chosen to be an entropy-conjugacy with a magic word. By symmetry, we obtain an entropy-conjugacy with a magic word $\sigma_{f} \rightarrow \sigma_{g}$. This finishes the proof in the mixing case.

Now suppose that $\sigma_{F}$ and $\sigma_{G}$ have period $p>1$. Write the $F, G$ in the form

$$
F(z)=\sum_{n=1}^{\infty} a_{n} z^{p n}, \quad G(z)=\sum_{n=1}^{\infty} b_{n} z^{p n} .
$$

Define $a=\sum a_{n} z^{n}$ and $b=\sum b_{n} z^{n}$. The loop shifts $\sigma_{a}$ and $\sigma_{b}$ are mixing and by the previous argument there is a loop shift $\sigma_{c}, c=\sum c_{n} z^{n}$, with injective one-block codes $\sigma_{c} \rightarrow \sigma_{a}$ and $\sigma_{c} \rightarrow \sigma_{b}$ with magic words. Let $H(z)=\sum c_{n} z^{p n}$. It follows easily that there are injective one-block codes $\sigma_{H} \rightarrow \sigma_{F}$ and $\sigma_{H} \rightarrow \sigma_{F}$ with magic words.

Theorem 6.4. Suppose $S_{A}$ and $S_{B}$ are Markov shifts, and $S_{A}$ is SPR with finite entropy. Then the following are equivalent:

(1) $S_{B}$ is $S P R, h\left(S_{A}\right)=h\left(S_{B}\right)$ and $\operatorname{period}\left(S_{A}\right)=\operatorname{period}\left(S_{B}\right)$.

(2) $S_{A}$ and $S_{B}$ are almost isomorphic.

(3) $S_{B}$ is $S P R, S_{A}$ and $S_{B}$ are entropy-conjugate.

Moreover, under these conditions there exists an entropy-conjugacy which is induced by an almost isomorphism.

ProOF: $(2) \Longrightarrow(1)$. The almost isomorphism induces a bijection respecting finitary isomorphism between the ergodic measures of $S_{A}$ and $S_{B}$ with full support. Therefore $S_{B}$ has a measure of maximal entropy $\mu_{B}$, and there is a finitary isomorphism $\left(S_{A}, \mu_{A}\right) \rightarrow\left(S_{B}, \mu_{B}\right)$. Because exponential recurrence is an invariant of finitary isomorphism, it follows from Proposition 2.3 that $S_{B}$ is SPR. The entropy and period of $S_{A}$ and $S_{B}$ are already invariants under measure-preserving isomorphism of $\left(S_{A}, \mu_{A}\right)$ and $\left(S_{B}, \mu_{B}\right)$.

This last remark also proves $(3) \Longrightarrow(1)$.

$(1) \Longrightarrow(2)$ and $(3)$. Pick a vertex $v$ in the graph with adjacency matrix $A$ which is used to define the edge shift $S_{A}$. Because $S_{A}$ has finite entropy, for each $n$ the number $F_{n}$ of first return loops to $v$ is finite. Let $F=\sum_{n=1}^{\infty} F_{n} z^{n}$. The natural injection $\sigma_{F} \rightarrow S_{A}$ is a one-block code with a magic word. Similarly choose $\sigma_{G}$ for $S_{B}$. It suffices to prove the theorem with $\sigma_{F}$ and $\sigma_{G}$ in place of $S_{A}$ and $S_{B}$.

Let $\log (\lambda)$ denote the entropy of $\sigma_{F}$ and $\sigma_{G}$. Because $S_{A}$ and $S_{B}$ are SPR, it follows from Corollary 4.4 that

$$
\varlimsup|| \mathcal{O}_{n}\left(\sigma_{F}\right)|-| \mathcal{O}_{n}\left(\sigma_{G}\right)||^{1 / n}<\lambda .
$$

Now Theorem 6.2 shows that $\sigma_{F}$ and $\sigma_{G}$ are almost isomorphic, by an almost isomorphism which induces an entropy-conjugacy.

Theorem 6.4 begs the following questions.

Questions 6.5. Is SPR an entropy-conjugacy invariant? On the other hand, must positive recurrent Markov shifts of equal entropy and period be entropy-conjugate? 


\section{Application to Other DYNAMiCAL SYSTEMS}

Given a measurable system $T$ (as defined in (3.2)), we begin by recalling the definition of the natural extension of $T$ (to an invertible system). Let $\bar{X}$ denote the subset of $X^{\mathbb{Z}}$ consisting of the bisequences $\left(\ldots x_{-1}, x_{0}, x_{1}, \ldots\right)$ such that $T\left(x_{i}\right)=x_{i+1}$ for all $i$. Associated to $\bar{X}$ is the $\sigma$-algebra generated by requiring every coordinate projection to be measurable. Let $\bar{T}$ be the bijection $\bar{X} \rightarrow \bar{X}$ defined by applying $T$ coordinatewise. The projection of $\bar{X}$ onto the first coordinate defines a map which intertwines $T$ and $\bar{T}$ (if $T$ is invertible, then this coordinate projection is a bijection). This coordinate projection induces an entropy-respecting bijection $\mathcal{M}(\bar{T}) \rightarrow \mathcal{M}(T)$ of invariant Borel probabilities. Often measurable phenomena are more conveniently studied on invertible systems, and the natural extension is used for this.

Now supose $\gamma$ is an entropy conjugacy of invertible systems. We say $\gamma$ refines the hyperbolic structure if the entropy-negligible set of Definition 3.3 can be chosen such that for $x, y$ in its complement,

$$
\begin{aligned}
\lim _{n \rightarrow \infty} \operatorname{dist}\left(\gamma\left(T^{n} x\right), \gamma\left(T^{n} y\right)\right) & =0 \Longrightarrow \lim _{n \rightarrow \infty} \operatorname{dist}\left(T^{n} x, T^{n} y\right)=0, \quad \text { and } \\
\lim _{n \rightarrow-\infty} \operatorname{dist}\left(\gamma\left(T^{n} x\right), \gamma\left(T^{n} y\right)\right) & =0 \Longrightarrow \lim _{n \rightarrow-\infty} \operatorname{dist}\left(T^{n} x, T^{n} y\right)=0 .
\end{aligned}
$$

We say $\gamma$ preserves the hyperbolic structure if both $\gamma$ and its inverse refine it.

Our next theorem is essentially an assembly of results from $[5,6,8,9]$, collected for the statement of Theorem 7.2. To prepare, recall some definitions. A piecewise monotonic interval map is a map $f:[0,1] \rightarrow[0,1]$ such that there is a finite partition of $[0,1]$ into intervals on each of which the restriction of $f$ is continuous and strictly monotonic. The map $f$ is said to be completely discontinuous if the previously mentioned partition can be chosen so that all its endpoints except 0 and 1 are discontinuities of $f$.

A multi-dimensional $\beta$-transformation $[6]$ is a map defined by $x \mapsto B . x \bmod \mathbb{Z}^{d}$ for $x \in[0,1]^{d}, d=1,2, \ldots$ and $B$ an expanding affine map of $\mathbb{R}^{d}$.

We will not define the class of subshifts of quasi finite type [9]; this is a useful class of finite state subshifts which contains the class of subshifts of finite type, but is much larger (for example, every finite positive entropy is achieved by a subshift of quasi finite type).

Theorem 7.1. The following measurable dynamical systems have natural extensions which are entropy-conjugate to the disjoint union of finitely many SPR Markov shifts of equal entropy.

(1) Subshifts of quasi finite type [9].

(2) Piecewise monotonic interval maps with non-zero topological entropy.

(3) The multi-dimensional $\beta$-transformations [6].

(4) $C^{\infty}$ smooth entropy-expanding maps including, e.g., smooth interval maps with non-zero topological entropy [5] or $(x, y) \mapsto\left(a-x^{2}+\epsilon y, b-y^{2}+\epsilon x\right)$ with $a, b<2$ close to 2 and $|\epsilon|$ sufficiently small $[7,8]$.

Moreover, the entropy-conjugacy from the natural extension to the SPR Markov shift can be chosen to refine the hyperbolic structure. In cases (1) and (3) and for completely discontinuous piecewise monotonic maps, the entropy-conjugacy can be chosen to preserve the hyperbolic structure. ${ }^{2}$

\footnotetext{
${ }^{2}$ In cases (2) and (4), it is the hyperbolic structure of the symbolic dynamics which is preserved.
} 
Proof sketch. (1) follows from [9, Theorem 3].

(2) follows from [9, Lemma 2].

(3) also follows from [9, Lemma 2].

(4) An argument similar to that in [9, Lemma 2] can be applied to $C^{\infty}$ smooth entropy-expanding maps in arbitrary dimension (see [8]). More specifically, in the proof in section III of [8], the condition that $\hat{\mu}\left(\Sigma_{*}\right)$ is zero can be replaced by the condition that it is very small. Then we can conclude that the Markov shift is SPR as in [9], Corollary at the end of section "Entropy at infinity".

The proof of the preservation of the hyperbolic structure is done in two steps. To begin, we recall that multidimensional $\beta$-transformations and completely discontinuous piecewise monotonic maps have natural extensions that are topologically conjugate to those of their symbolic dynamics. These are subshifts of quasi-finite type (or Q.F.T.) as shown in [9]. So we can restrict to Q.F.T.

We use terminology and facts from [9]. A Markov shift (the so-called complete Markov diagram) is built over the Q.F.T. Let $\gamma$ be the entropy-conjugacy of the original subshift into that Markov shift. $\gamma$ obviously refines the hyperbolic structure as the canonical partition of the Markov shift is finer than the original canonical partition, using the identification $\gamma$.

On the other hand, for $x$ in the Markov shift, $x_{0}$ is determined by the sequence $\left(\gamma^{-1} x\right)_{n}, n \leq 0$. Thus the unstable relation is preserved by $\gamma$.

One cannot replace $n \leq 0$ by $n \geq 0$ here. But we have the following property for a.e. point $x$ in the Markov shift: $x_{n}$ is determined by the finite word $(\gamma x)_{k}, n-\ell \leq$ $k \leq n$ with $\ell=\ell\left(\sigma^{n} x\right)$. By stationarity $\ell\left(\sigma^{n} x\right) \nrightarrow \infty$ for a.e. $x$. Hence the future in the subshift determines $x_{n_{0}}$ for some $n_{0}$. But $x_{n_{0}}$ and the sequence $\left(\gamma^{-1} x\right)_{k}, k \geq n_{0}$ determine $x_{k}, k \geq n_{0}$. Thus the stable relation is also preserved by $\gamma$. This concludes the proof for the case of Q.F.T.

Combining Theorem 7.1 with our main result, Theorem 6.4, we obtain

Theorem 7.2. A system in any of the classes listed in Theorem 7.1 has finite entropy and only finitely many ergodic measures of maximal entropy. Two systems from the list have natural extensions which are entropy conjugate if and only if they have equal entropy and for each $p \in \mathbb{N}$ the same number of ergodic maximal entropy measures of period $p$.

Corollary 7.3. Two topologically mixing piecewise monotonic interval maps are entropy conjugate if and only if they have the same entropy.

Proof. A topologically mixing piecewise monotonic map has a unique measure of maximal entropy, and this measure is mixing [26]. So, in this case the invariant above simplifies to the entropy of that measure, which is the topological entropy by the variational principle [63].

\section{REMARKS}

We continue our convention that "Markov shift" means "irreducible Markov shift", even in the finite state case.

Remark 8.1 (Magic words). Magic words come from the finite state coding theory of Markov shifts [31, 35]. They play a basic role in the structure theory of finite-toone codes between finite-state Markov shifts (where they are defined more generally for maps which are finite-to-one but not necessarily one-to-one almost everywhere). 
In the past, a major step in some code constructions was to show choices could be made to guarantee existence of a magic word, so that the resulting code would be one-to-one almost everywhere. (For example, the construction of a magic word is the essential difference between [1] and [41].) Ashley's Replacement Theorem $[31,35]$ has largely eliminated this step for codes of finite state systems.

Remark 8.2 (Almost conjugacy). [1,31,35] Two finite state Markov shifts $S, T$ are almost conjugate [35], equivalently almost topologically conjugate [1,31], if there is another Markov shift $R$ and surjective block codes $R \rightarrow S, R \rightarrow T$ each of which are one-to-one almost everywhere. The Adler-Marcus Theorem [1] is that entropy and period are complete invariants of almost topological conjugacy for finite state Markov shifts. (Here each of the maps $R \rightarrow S, R \rightarrow T$ must have a magic word, and the content of "a.e." contains no more information than we have in Propositions 3.1 and 3.4.)

The classification of countable state Markov shifts up to almost conjugacy is extremely difficult and remains an open problem [12]. Doris Fiebig has the most general result: for Markov shifts $S, T$ of equal finite positive entropy, there is a Markov shift $R$ of equal entropy and countable-to-one biclosing continuous surjections $R \rightarrow S, R \rightarrow T$. Here "biclosing" is a bonus and it is impossible to replace "countable-to-one" with "uniformly finite-to-one" [12]. Note, this result places no restriction on the relative classes of $S$ and $T$ (which can be transient, null recurrent, positive recurrent or strong positive recurrent, each independent of the other).

Remark 8.3 (Almost isomorphism). Our definition of "almost isomorphism" is analogous to the definition of "almost conjugacy"; for countable state Markov shifts, it seems more tractable and perhaps better suited to studying invariant measures. Injective block codes between finite state Markov shifts of equal entropy must be homeomorphisms, so "almost isomorphism" does not appear as a meaningful distinct relation in the finite state category.

Remark 8.4 (Other Markov measures). The Adler-Marcus Theorem gave good finitary codes between finite state Markov shifts of equal entropy and period. By a Markov chain, we will mean a Markov shift together with an invariant Markov measure. The subsequent search for good finitary codes between finite state Markov chains has a sizeable history (see [37, 39, 42, 43, 53, 54, 55] and their references); for years, there was an attempt to produce general codes by way of an almost topological conjugacy. Marcus and Tuncel destroyed these hopes with their weightper-symbol polytope invariants [37]. The paper [39] of Mouat and Tuncel effectively exploits one alternate approach, using ordered algebra. The "loops method" of this paper is another approach; we hope it will be of use for Markov chains beyond the case of the measure of maximal entropy. Both the Mouat-Tuncel construction and the Loops Lemma method yield magic word isomorphisms when they work. We expect that the known invariants (ratio group [33], weights group with distinguished coset [43], beta function [53]) of hyperbolic-structure-preserving isomorphism $[54,55]$ with finite-expected coding time will be the only obstructions to the existence of a magic word isomorphism between finite state mixing Markov chains.

Remark 8.5 (Local compactness and loop shifts). Where we use "irreducible Markov shift", Gurevich and Savchenko [24] use "indecomposable symbolic Markov chain". Their use of "symbolic" rather than "topological" was to emphasize that topological 
issues are not always paramount. The following simple construction illustrates this point in our context.

Let $\sigma_{f}$ be any loop shift such that $f(1)=\infty$ (i.e., $f$ is not just a polynomial). Then the space $\sigma_{f}$ will be neither locally compact nor $\sigma$-compact. The loop shift is still closely related to a locally compact system. The graph in Figure 1 describes the construction of a locally compact $S_{A}$ from $f$, such that the natural injective one block code $\sigma_{f} \rightarrow S_{A}$ has many magic words and induces a bijection of spaces of invariant Borel probabilities, $\mathcal{M}\left(\sigma_{f}\right) \rightarrow \mathcal{M}\left(S_{A}\right)$.

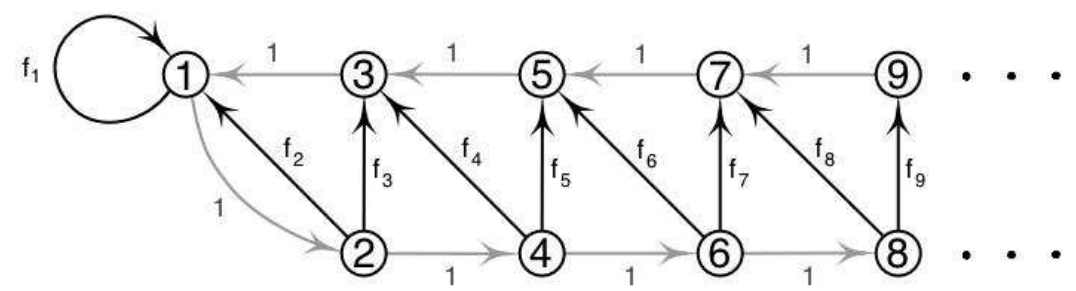

Figure 1.

\section{REFERENCES}

[1] R. Adler and B. Marcus, Topological entropy and equivalence of dynamical systems, Memoirs Amer. Math. Soc. 20 (1979), no. 219.

[2] L. Block, J. Guckenheimer, M. Misiurewicz and L.S. Young, Periodic points and topological entropy of one-dimensional maps, Global theory of dynamical systems, pp. 18-34, Lecture Notes in Math. 819 (1980), Springer-Verlag.

[3] R. Bowen, Equilibrium states and the ergodic theory of Anosov diffeomorphisms, Lecture Notes in Math. 470 (1975), Springer-Verlag.

[4] M. Boyle, J. Buzzi and R. Gomez, Good potentials for almost isomorphism of countable state Markov shifts, preliminary manuscript, 2004.

[5] J. Buzzi, Intrinsic ergodicity of smooth interval maps, Israel J. Math. 100 (1997), 125161.

[6] J. Buzzi, Intrinsic ergodicity of affine maps on [0, 1] $]^{d}$, Monat. Math. 124 (1997), 97-118.

[7] J. Buzzi, Ergodicité intrinsèques de produits fibrés d'applications chaotiques unidimensionnelles, Bull. Soc. Math. France 126 (1998), 51-77; English preprint version at http://math.polytechnique.fr/cmat/buzzi.

[8] J. Buzzi, On entropy-expanding maps, preprint CMAT, 2000.

[9] J. Buzzi, Subshifts of quasi-finite type, preprint CMAT, 2003.

[10] J. Buzzi, O. Sarig, Uniqueness Of Equilibrium Measures For Countable Markov Shifts And Multidimensional Piecewise Expanding Maps, Ergod. Th. \& Dynam. Syst. 23 (2003), $1383-1400$.

[11] E.E.Cummings, Collected works 1913-1962, Harcourt Brace Jovanovich, Inc. (1972).

[12] D. Fiebig, Common extensions for locally compact Markov shifts, Monatsh. Math. 132 (2001), no. 4, 289-301.

[13] D. Fiebig, Graphs with pre-assigned Salama entropies and optimal degrees for locally compact Markov shifts, Ergodic Theory Dynam. Systems 23 (2003), no. 4, 1093-1124.

[14] D. Fiebig, Factor theorems for locally compact Markov shifts Forum Math. 14 (2002), no. $4,623-640$.

[15] D. Fiebig, Factor maps, entropy and fiber cardinality for Markov shifts, Rocky Mountain J. Math. 31 (2001), no. 3, 955-986.

[16] D. Fiebig and U. Fiebig, Entropy and finite generators for locally compact subshifts, Ergodic Theory Dynam. Systems 17 (1997), no. 2, 349-368.

[17] D. Fiebig, U.-F. Fiebig and M. Yuri, Pressure and equilibrium states for countable state Markov shifts, Israel J. Math. 131 (2002), 221-257.

[18] U.-R. Fiebig, A return time invariant for finitary isomorphisms, Ergodic Theory Dynam. Systems 4 (1984), no. 2, 225-231. 
[19] U.-R. Fiebig, Symbolic dynamics and locally compact Markov shifts, 1996. Habilitationsschrift, U. Heidelberg.

[20] R. Gomez, Positive K-theory for finitary isomorphisms of Markov chains, Ergodic Theory Dynam. Systems 23 (2003), pp 1485-1504.

[21] B. M. Gurevich, Shift entropy and Markov measures in the path space of a denumerable graph (Russian), Dokl. Akad. Nauk SSSR 187 (1969), 715-718; English translation: Soviet Math. Dolk. 10, 4, 911-915.

[22] B. M. Gurevich, Stably recurrent nonnegative matrices (Russian), Uspekhi Mat. Nauk 51 (1996), no. 3(309), 195-196; translation in Russian Math. Surveys 51 (1996), no. 3, $551-552$.

[23] B. M. Gurevich and A.B. Polyakov, Stably recurrent functions on the loop space of a countable graph, Uspekhi Mat. Nauk 54 (1999), no. 6(330), 157-158; translation in Russian Math. Surveys 54 (1999), no. 6, 1242-1243.

[24] B. M. Gurevich and S. Savchenko, Thermodynamical formalism for symbolic Markov chains with a countable number of states (Russian), Uspekhi Mat. Nauk 53 (1998), 3106; translation in Russian Math. Surveys 53 (1998), 245-344.

[25] F. Hofbauer, $\beta$-shifts have unique maximal measure, Monatsh. Math. 85 (1978), no. 3, 189-198.

[26] F. Hofbauer, On intrinsic ergodicity of piecewise monotonic transformations with positive entropy I, Israel J. Math. 34 (1979), no. 3, 213-237; II Israel J. Math. 38 (1981), no. 1-2, $107-115$.

[27] F. Hofbauer, Piecewise invertible dynamical systems, Probab. Theory Relat. Fields 72 (1986), no. 3, 359-386.

[28] M. Keane and M. Smorodinsky, Finitary isomorphisms of irreducible Markov shifts, Israel J. Math. 34 (1979), no. 4, 281-286 (1980).

[29] G. Keller, Markov extensions, zeta functions, and Fredholm theory for piecewise invertible dynamical systems, Trans. Amer. Math. Soc. 314 (1989), no. 2, 433-497.

[30] G. Keller, Lifting measures to Markov extensions, Monatsh. Math. 108 (1989), no. 2-3, 183-200.

[31] B.P. Kitchens, Symbolic dynamics. One-sided, two-sided and countable state Markov shifts, Springer-Verlag (1998).

[32] U. Krengel, Ergodic theorems, Walter de Gruyter, (1985).

[33] W. Krieger, On the finitary isomorphisms of Markov shifts that have finite expected coding time, Z. Wahrsch. Verw. Gebiete 65 (1983), no. 2, 323-328.

[34] F. Ledrappier, Principe variationnel et systèmes dynamiques symboliques, Z. Wahrsch. Verw. Gebiete 30 (1974), 185-202.

[35] D. Lind and B. Marcus, An introduction to Symbolic Dynamics and Coding, Cambridge University Press (1995).

[36] C. Liverani, B. Saussol, S. Vaienti, Conformal measure and decay of correlation for covering weighted systems, Ergodic Theory Dynam. Systems 18 (1998), 1399-1420.

[37] B. Marcus and S. Tuncel, The weight-per-symbol polytope and scaffolds of invariants associated with Markov chains, Ergodic Theory Dynam. Systems 11 (1991), 129-180.

[38] R. D. Mauldin and M. Urbański, Gibbs states on the symbolic space over an infinite alphabet. Israel J. Math. 125 (2001), 93-130.

[39] R. Mouat and S. Tuncel, Constructing finitary isomorphisms with finite expected coding times, Israel J. Math. 132 (2002), 359-372.

[40] D. Ornstein, Bernoulli shifts with the same entropy are isomorphic. Advances in Math. 4 (1970), 337-352 (1970).

[41] W. Parry, A finitary classification of topological Markov chains and sofic systems, Bull. London Math. Soc. 9 (1977), no. 1, 86-92.

[42] W. Parry, Notes on coding problems for finite state processes, Bull. London Math. Soc.23 (1991), 1-33.

[43] W. Parry and K. Schmidt, Natural coefficients and invariants for Markov shifts, Inventiones Math. 76 (1984), 15-32.

[44] D. Rudolph, A mixing Markov chain with exponentially decaying return times is finitarily Bernoulli, Ergodic Theory Dynam. Systems 2 (1982), no. 1, 85-97.

[45] S. Ruette, On the Vere-Jones classification and existence of maximal measures for countable topological Markov chains, Pacific J. Math. 209 (2003), 365-380 
[46] I. Salama, Topological entropy and recurrence of countable chains, Pacific J. Math. 134 (1988), no. 2, 325-341; corrections 140 (1989), no. 2, 397.

[47] I. Salama, On the recurrence of countable topological Markov chains, in Symbolic dynamics and its applications (New Haven, CT, 1991), Contemp. Math., 135 (1992), 349-360, Amer. Math. Soc., Providence, RI,

[48] O. Sarig, Thermodynamic formalism for countable Markov shifts, Ergodic Theory Dynam. Systems 19 (1999), no. 6, 1565-1593.

[49] O. Sarig, Thermodynamic formalism for null recurrent potentials, Israel J. Math. 121 (2001), 285-311.

[50] O. Sarig, Phase transitions for countable Markov shifts, Comm. Math. Phys. 217 (2001), no. $3,555-577$.

[51] O. Sarig, Subexponential decay of correlations, Invent. Math. 150 (2002), 629-653.

[52] O. Sarig, Existence of Gibbs measures for countable Markov shifts, Proc. Amer. Math. Soc. 131 (2003), no. 6, 1751-1758

[53] K. Schmidt, Invariants for finitary isomorphisms with finite expected code lengths, Invent. Math. 76 (1984), no. 1, 33-40.

[54] K. Schmidt, Hyperbolic structure preserving isomorphisms of Markov shifts, Israel J. Math. 58 (1987), no. 2, 225-242.

[55] K. Schmidt. Hyperbolic structure preserving isomorphisms of Markov shifts, Israel J. Math. 55 (1986), no. 2, 213-228.

[56] Y. Takahashi, Isomorphisms of $\beta$-automorphisms to Markov automorphisms, Osaka J. Math. 10 (1973), 175-184.

[57] Y. Takahashi, Shift with orbit basis and realization of one dimensional maps, Osaka J. Math. 20 (1983), 599-629; Correction Osaka J. Math. 22 (1985), 637.

[58] M. Thaler, Estimates of the invariant densities of endomorphisms with indifferent fixed points, Israel J. Math. 37 (1980), 303-314.

[59] M. Urbański and A. Zdunik, Hausdorff dimension of harmonic measure for self-conformal sets, Adv. Math. 171 (2002), no. 1, 1-58.

[60] D. Vere-Jones, Ergodic properties of non-negative matrices, Pacific J. Math. 22 (1967), $361-386$.

[61] D. Vere-Jones, Geometric ergodicity in denumerable Markov chains, Quart. J. Math. Oxford (2) 13 (1962), 7-28.

[62] P. Walters, Ruelle's operator theorem and g-measures, Trans. Amer. Math. Soc. 214 (1975), 375-387.

[63] P. Walters, An introduction to ergodic theory, Springer-Verlag (1982).

[64] L.-S. Young, Recurrence times and rates of mixing, Israel J. Math. 110 (1999), 153-188.

[65] M. Yuri, On the convergence to equilibrium states of certain non-hyperbolic systems, Ergod. Th. \& Dynam. Syst. 17 (1997), 977-1000.

Mike Boyle, Department of Mathematics, University of Maryland, College Park, MD 20742-4015, U.S.A.

E-mail address: mmb@math.umd.edu

$U R L$ : www. math.umd.edu/ mmb

Jerome Buzzi, Centre de Mathématiques, Ecole polytechnique, 91128 Palaiseau Cedex, FRANCE

E-mail address: buzzi@math.polytechnique.fr

$U R L$ : www.geocities.com/jrbuzzi/

Ricardo Gomez, Instituto de Matematicas Area de la Investigacion Cientifica, CirCuito Exterior, Ciudad Universitaria, DF 04510, Mexico

E-mail address: rgomez@math.unam.mx

$U R L$ : www. math. unam.mx/ rgomez 